\title{
Introduction of the special issue "How does the prostate cancer microenvironment affect the metastatic process and/or treatment outcome?"
}

\author{
Jason P. Webber ${ }^{1}$, Klaus Pors ${ }^{2}$ \\ ${ }^{1}$ Tissue Microenvironment Group, Division of Cancer \& Genetics, School of Medicine, Tenovus Building, Cardiff University, Cardiff CF14 4XN, UK. \\ ${ }^{2}$ Institute of Cancer Therapeutics, Faculty of Life Sciences, University of Bradford, Bradford BD7 1DP, UK.
}

Correspondence to: Dr. Jason P. Webber, Tissue Microenvironment Group, Division of Cancer \& Genetics, School of Medicine, Tenovus Building, Cardiff University, Cardiff CF14 4XN, UK. E-mail: WebberJP@Cardiff.ac.uk; Dr. Klaus Pors, Institute of Cancer Therapeutics, Faculty of Life Sciences, University of Bradford, Bradford BD7 1DP, UK. E-mail: k.pors1@bradford.ac.uk

How to cite this article: Webber JP, Pors K. Introduction of the special issue "How does the prostate cancer microenvironment affect the metastatic process and/or treatment outcome?". J Cancer Metastasis Treat 2018;4:1-3.

Article history: Received: 14 Jul 2018 Accepted: 16 Jul 2018 Published: 21 Aug 2018

It is with great pleasure that we introduce this special issue titled "How does the prostate cancer microenvironment affect the metastatic process and/ or treatment outcome?". Within this issue we, and our fellow authors, explore the role of the prostate cancer microenvironment in tumour metastasis and treatment outcome. Global statistics reveal that prostate cancer is the second most common form of cancer and is attributable to fifth of all cancer-related deaths to affect men worldwide. Whilst rates of disease incidence appear to be increasing a high proportion of men diagnosed with disease will survive for ten or more years. Yet, for some men, the disease is far more aggressive, resulting in tumour metastasis and failed response to treatment. Improving our knowledge of the prostate cancer microenvironment will undoubtedly lead to opportunities for providing better treatment options for patients with aggressive forms of this disease. To address this gap in knowledge, we here present nine articles detailing the current state of art regarding development of aggressive prostate cancer.

Current methods of prostate cancer diagnosis lack both sensitivity and specificity. This can result in failed diagnosis of men likely to develop aggressive disease and over-treatment of men who do not require treatment who consequently suffer as a result. It is clear that if we are to improve the current paradigm clinicians and scientists must work together. The first and the last articles in this special issue, written by Mason[1] and Maitland[2], explores how to improve on preclinical models and collaboration between scientists and clinicians in combined efforts towards improving the quality of lives of patients living with prostate cancer.

With our improved understanding of the prostate cancer development, it is clear that our attitudes towards cancer diagnosis and therapeutic intervention need to evolve

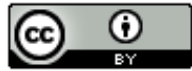

This is an open access article licensed under the terms of Creative Commons Attribution 4.0 International License (https://creativecommons.org/licenses/by/4.0/), which permits unrestricted use, distribution, and reproduction in any medium, as long as the original author is credited and the new creations are licensed under the identical terms.

For reprints contact: service@oaepublish.com
}

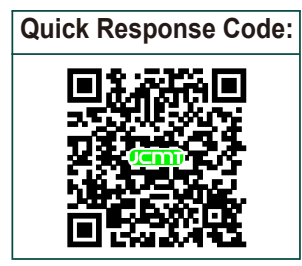


also. Researchers require better models that more accurately represent the tumour microenvironment, but this requires collaboration between basic scientists and clinicians. Only by working together can we truly hope to develop personalised, effective, cancer therapies and therefore improve the survival of men with prostate cancer. The two commentaries by Mason ${ }^{[1]}$ and Maitland ${ }^{[2]}$ encapsulate seven in-depth reviews, which are focussed on key aspects that contribute to prostate cancer aggressiveness.

Perhaps one of the most studied aspects of prostate cancer is the role of androgens. The prostate requires androgens, a family of hormones including testosterone, which interact with the androgen receptor $(A R)$ to regulate normal physiological function. Aberrant androgen signalling, however, can drive the formation and growth of prostatic tumours. Androgen deprivation therapy therefore remains a pivotal treatment for prostate cancer. Abiraterone and enzalutamide are two such therapeutic agents used to treat prostate cancer. Regrettably, tumours can become resistant to such therapies, and there is urgent need for new therapies to improve patient survival. Pippione et al. ${ }^{[3]}$ review the androgen-AR axis and the potential targeting of steroidogenic enzymes as a means of overcoming resistance to existing prostate cancer treatments.

Cancer progression is associated with a dysregulated balance between cellular growth, division, and cell death. Such processes are regulated by transcription factors that work alone, or in combination with other proteins, to regulate genetic expression. One family of transcription factors, the HOX proteins, have been shown to contribute to interactions between prostate tumours and the surrounding microenvironment. Morgan and Pandha ${ }^{[4]}$ review the numerous roles of HOX proteins in prostate cancer, ranging from regulation of androgen-receptor sensitivity to angiogenesis and tumour metastasis.

Tumour growth within the prostate, and other sites, requires remodelling of the surrounding environment. Matrix metalloproteinases (MMPs), a family of proteolytic enzymes, have long been associated with regulation of the extracellular matrix and tissue remodelling. They have also been implicated in the initiation, progression and metastasis of multiple cancer types, including prostate cancer. The MMP family is composed of two broad sub-groups, the soluble or secreted MMPs and the membrane-type MMPs (MT-MMPs). Whilst many studies have focussed on the soluble MMPs, the expression and roles of MT-MMPs remain less clear and form the subject of review by Falconer and Loadman ${ }^{[5]}$. Furthermore, the authors consider MT-MMP expression and proteolytic capacity in the design of potential new therapies against metastatic prostate cancer.

Cell to cell communication plays a key role in the development of prostate cancers. As is evident from numerous articles within this special issue, such communication occurs not only between cancer cells but also between all cells within the tumour microenvironment, resulting in changes to the local environment that favour tumour growth and metastasis. Historically, many studies have focussed on soluble growth factors and cytokines, but there is emerging evidence highlighting the role of extracellular vesicles. Such vesicles can be divided into two broad sub-categories, microvesicles and exosomes. Whilst there have been many studies exploring the role of extracellular vesicles in cancer, relatively few of these studies have focussed on prostate cancer. Shephard et al. ${ }^{[6]}$ review the reported functions of extracellular vesicles from diverse malignancies to identify those with potential relevance to prostate cancer. It is clear that extracellular vesicles represent a means of delivering a complex assortment of factors from one cell to another, actively contributing to the disease progress. Such vesicles also represent an attractive source of biomarkers for both diagnostic and prognostic purposes.

Despite the wealth of knowledge on prostate cancer and potential therapeutic interventions there remain many unanswered questions and challenges to address. One such area is that of tumour heterogeneity. Heterogeneity within prostatic tumours is a complex topic and forms the basis of an original research article by Frame et al. ${ }^{[7]}$. When we think of heterogeneity we should not only consider patient variability, but also differences between individual tumours and subpopulations of cells within a tumour. Not only do the authors consider how to tackle tumour heterogeneity, but they introduce a new model for assessing drug response that takes into account this heterogeneity. As an extension of tumour heterogeneity, McKenna et al. ${ }^{[8]}$ discusses how the presence of hypoxia, which is wellknown to contribute to cancer aggressiveness and resistance to chemo- and radiotherapy, can be turned into an opportunity for rationalised drug discovery and combination therapy while Ibrahim et al. ${ }^{[9]}$ discusses how tumour-initiating cells with high expression of aldehyde dehydrogenase contribute to treatment resistance and tumour recurrence.

We would like to thank all of the contributing authors for their hard work in producing the enclosed articles. 
We hope you enjoy this special issue of the Journal of Cancer Metastasis and Treatment.

\section{DECLARATIONS}

Authors' contribution

Manuscript writting and revision: Webber JP, Pors K

\section{Availability of data and materials}

Not applicable.

\section{Financial support and sponsorship}

None.

\section{Conflicts of interest}

The authors declare there are no conflicts of interest.

\section{Ethical approval and consent to participate}

Not applicable.

\section{Consent for publication}

Not applicable.

\section{Copyright}

(c) The Author(s) 2018.

\section{REFERENCES}

1. Mason M. Getting better at treating prostate cancer: what clinicians should want from scientists. J Cancer Metastasis Treat 2017;3:271-7.

2. Maitland NJ. Getting closer to prostate cancer patients: what scientists should want from clinicians. J Cancer Metastasis Treat 2017;3:262-70.

3. Pippione AC, Boschi D, Pors K, Oliaro-Bosso S, Lolli ML. Androgen-AR axis in primary and metastatic prostate cancer: chasing steroidogenic enzymes for therapeutic intervention. $J$ Cancer Metastasis Treat 2017;3:328-61.

4. Morgan R, Pandha HS. HOX transcription factors and the prostate tumor microenvironment. J Cancer Metastasis Treat 2017:3:278-87.

5. Falconer RA, Loadman PM. Membrane-type matrix metalloproteinases: expression, roles in metastatic prostate cancer progression and opportunities for drug targeting. J Cancer Metastasis Treat 2017;3:315-27.

6. Shephard AP, Yeung V, Clayton A, Webber JP. Prostate cancer exosomes as modulators of the tumor microenvironment. $J$ Cancer Metastasis Treat 2017;3:288-301.

7. Frame FM, Noble AR, Klein S, Walker HF, Suman R, Kasprowicz R, Mann VM, Simms MS, Maitland NJ. Tumor heterogeneity and therapy resistance - implications for future treatments of prostate cancer. J Cancer Metastasis Treat 2017;3:302-14.

8. McKenna DJ, Errington R, Pors K. Current challenges and opportunities in treating hypoxic prostate tumors. $J$ Cancer Metastasis Treat 2018;4:11.

9. Ibrahim AIM, Sadiq M, Frame FM, Maitland NJ, Pors K. Expression and regulation of aldehyde dehydrogenases in prostate cancer. $J$ Cancer Metastasis Treat 2018;4:44 